\title{
Ejercicios físicos diarios de intensidad moderada en el interior del aula mejora la fuerza de tren inferior y la agilidad en escolares: un estudio cuasi experimental
}

Daily moderate-intensity physical exercises inside the classroom improves lower body strength and agility in schoolchildren: a quasi-experimental study

*,**Gaston Eduardo Ibañez Rojas, ***Ivana Leao Ribeiro, *Patricio Andres Inostroza Dominguez, *Daniela

Margarita Vergara Rojas, ***Ricardo Souza de Carvalho

*Centro de Formación Técnica SantoTomas (Chile), **Universidad SantoTomás (Chile), ***Universidad Católica del Maule (Chile)

Resumen. El estudio tuvo como objetivo determinar los cambios en la condición física y en el índice de masa corporal que se producen en una intervención de 16 semanas en escolares en el interior de la aula. Setenta y tres escolares ( $\mathrm{n}=34$ niñas; n=39 niños) con edad entre los 8 y 9 años recibieron las siguientes evaluaciones: flexibilidad de la musculatura dorsal de espalda (mediante la prueba de weels), fuerza muscular (mediante la prueba pararse y sentarse), agilidad (mediante la prueba 4x10m shuttle run) y índice de masa corporal (IMC) utilizando un estadiómetro y una balanza. Se aplicó un programa de entrenamiento con ejercicios de flexibilidad, fuerza y resistencia (30 minutos, 5x/semana). Hubo una disminución del IMC (diferencia de $0.60 \mathrm{~kg} / \mathrm{cm}^{2}$ en ambos géneros), aumento de la fuerza (10.4 repeticiones en niñas y 11.6 en niños) y mejora de la agilidad ( 0.91 segundos en niñas y 0.71 segundos en niños: $p<0.05$ en todas las comparaciones y tamaño de efecto entre 0.20-1.77 en niñas y 0.18-2.34 en niños). El índice de obesidad y sobrepeso entre el alumnado de la clase se vio reducido en un $5.3 \%$ y $5.9 \%$, respectivamente. No hubo diferencias respecto a la flexibilidad ( $\mathrm{p}>0.05$ ). Un entrenamiento diario en el interior del aula sin implementación y en un espacio de un metro cuadrado disminuye el IMC, mejora la fuerza de tren inferior y la agilidad en escolares.

Palabras-clave: actividad física, flexibilidad, fuerza muscular, índice de masa corporal, resistencia física, preescolar.

Abstract. The objective of the study was to determine the changes in physical condition and body mass index that occur in a 16-week intervention in schoolchildren inside the classroom. Seventy-three schoolchildren ( $\mathrm{n}=34$ girls; $\mathrm{n}=39$ boys) between the ages of 8 and 9 received the following evaluations: flexibility of the dorsal back muscles, through the weels test, muscular strength, through the standing and sitting test, agility, through the $4 \times 10 \mathrm{~m}$ shuttle test run and body mass index (BMI) using a stadiometer and scale. A training program was applied with flexibility, strength and resistance exercises were performed (30 minutes, $5 \mathrm{x} /$ week). There was a decrease in BMI, (difference of $0.60 \mathrm{~kg} / \mathrm{cm}^{2}$ in both genders), increase in strength $(10.4$ repetitions in girls and 11.6 in boys) and improvement of the agility ( 0.91 seconds in girls and 0.71 seconds in boys), $\mathrm{p}<0.05$ in all comparisons and effect size between 0.20-1.77 in girls and 0.18-2.34 in boys. The rate of obesity and overweight among the students in the class was reduced by one $5.3 \%$ and $5.9 \%$ respectively. There were no differences in flexibility $(\mathrm{p}>0.05)$. A daily training in schoolchildren without implementation and in a space of one square meter decreases BMI, improves lower body strength and agility in schoolchildren.

Key words: physical activity, flexibility, muscle strength, body mass index, physical endurance, preschool.

\section{Introducción}

El sobrepeso y la obesidad se definen como una acumulación anormal o excesiva de grasa que puede ser perjudicial para la salud (WHO, 2007). En Chile, la obesidad es considerada como un problema de salud pública principalmente durante los primeros años de vida (Mönckeberg \& Muzzo, 2015). Estudios anteriores han evidenciado la asociación de obesidad en la edad preescolar (Al-Muhaimeed, Dandash, Ismail, \& Saquib,

Fecha recepción: 30-07-21. Fecha de aceptación: 01-12-21

Ricardo Souza de Carvalho

ricardosocar@gmail.com
2015; Carsley, Tu, Parkin, Pullenayegum, \& Birken, 2018). Según la Organización Mundial de Salud (2016), se considera sobrepeso cuando el índice de masa corporal (IMC) para la edad se presenta con más de una desviación típica y por encima de la mediana establecida en los patrones de crecimiento infantil. Por otro lado, se considera obesidad cuando hay más de dos desviaciones típicas por encima de la mediana establecida (WHO, 2016).

Según la Organización mundial de la Salud, la causa fundamental del sobrepeso y la obesidad es producto de un desequilibrio energético entre calorías consumidas y gastadas. En Chile, el 15.2\% de niños y adolescentes (entre 5 y 19 años) presentaron obesidad y cerca de 
$38.3 \%$ de niños y niñas con edad entre 5 y 9 años presentaron sobrepeso (WHO, 2016). A nivel mundial ha ocurrido un aumento en la ingesta de alimentos de alto contenido calórico que son ricos en grasas y un descenso en la actividad física debido a la naturaleza cada vez más sedentaria de la población (Tambalis, Panagiotakos, Psarra, \& Sidossis, 2018), definiendo sedentarismo como falta de actividad física realizada de manera sistemática (Carson et al., 2016).

Junto con lo anterior, la práctica de la actividad física parece ser una de las fórmulas más efectiva para prevenir la obesidad en niños de edad preescolares en Chile (Cruz et al., 2016; Giakoni, Bettancourt, \& DuclosBastías, 2021; Rodríguez-Fernández, Rico-Díaz, NeiraMartín, \& Navarro-Patón, 2021). Escolares con mayor capacidad aeróbica han presentado mayor rendimiento académico(Rosa Guillamón, Garcia Canto, \& Jose Carrillo López, 2019)y los niños suelen practicar más actividades físicas que las niñas dentro y fuera del contexto escolar(Rodríguez-Fernández et al., 2021). A nivel internacional la evidencia apunta que las clases de educación física no cumplen con la intensidad mínima necesaria de ejercicios moderados a vigorosos recomendados para escolares(Hollis et al., 2017) para mejorar los indicadores de salud sobre obesidad y sedentarismo. En este contexto, solamente un estudio fue realizado en el interior de las escuelas, con una duración promedio de 6 minutos y 3 veces a la semana con la modalidad de ejercicio utilizando intervalos a altas intensidades(Engel et al., 2019), indicando que el entrenamiento realizado fue beneficioso para aumentar la fuerza muscular. Sin embargo, todavía faltan estudios que involucren la participación de las familias y personas más cercanas a estos niños, para fomentar los hábitos hacia la actividad física diaria en el interior del aula, para cumplir con las directrices de la Organización Mundial de Salud en cuanto a la práctica diaria de ejercicios.

En este contexto, considerando el rol que ejerce la escuela en la influencia de nuestros niños y niñas, se pretende realizar un estudio en el interior del aula, el cual consiste en implementar ejercicios de intensidad moderada, en espacio reducidos y sin materiales deportivo de apoyo, con el objetivo de conocer los cambios en la condición física y el índice de masa corporal, que estos ejercicios provocan en los niños que cursan el tercer año básico. El objetivo del estudio fue determinar los cambios en ciertos aspectos de la condición física y en el índice de masa corporal que se producen en una intervención de 16 semanas en escolares al interior del aula.

\section{Métodos}

\section{Diseño de investigación}

Corresponde a un diseño experimental del tipo cuasi experimental, debido a que los grupos del estudio no fueron asignados al azar (Bärnighausen, Røttingen, Rockers, Shemilt, \& Tugwell, 2017) y de corte longitudinal. El muestreo es intencional, por conveniencia y no probabilístico, debido a que los casos no fueron seleccionados aleatoriamente de la población (Bornstein, Jager, \& Putnick, 2013).

\section{Participantes}

La población escogida para el estudio fueron estudiantes de tres grupos de escolares por conveniencia (tercero año básico A, B y C) de una Escuela Básica de la comuna de Colbún. Al ser menores de edad los participantes y apoderados fueron orientados sobre los objetivos y procedimientos de la investigación. Cuando los padres consintieron, los estudiantes podrían rechazar participar del proyecto. Aquellos que no desearon participar de los ejercicios se mantuvieron en su puesto hasta el cierre de la actividad.

El estudio fue aprobado por el comité de ética científico de la Universidad Santo Tomás, (número 154/ 2019) y todos los involucrados fueron guiados acerca de los objetivos y procedimientos de la investigación, así como los riesgos y beneficios.

Junto con lo anterior, ochenta y cuatro estudiantes fueron reclutados para participar del estudio y 73 participaron de todas las etapas del estudio. La figura 1 presenta el flujograma de los participantes.

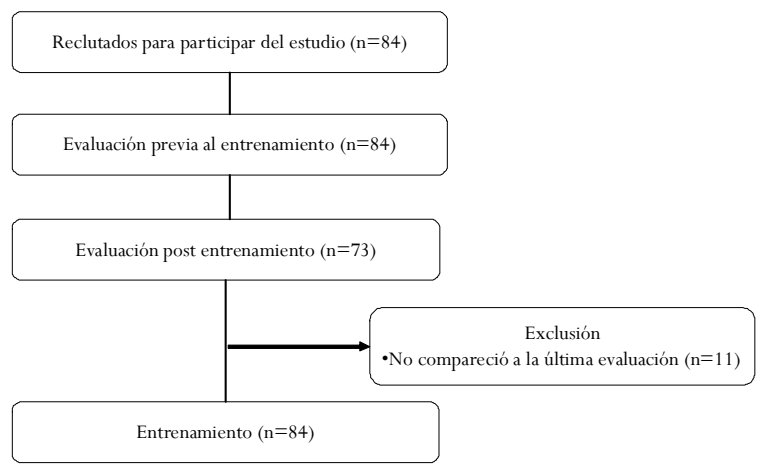

Figura 1. Flujograma de los participantes del estudio

\section{Criterios de inclusión}

Fueron incluidos niños y niñas que estaban cursando el tercer año básico regularmente matriculados en una escuela básica de la comuna de Colbún, con edades entre 8 y 9 años. 


\section{Criterios de exclusión}

Los niños o niñas que presentaron algún diagnóstico previo de patología crónica o lesiones musculo esqueléticas que imposibilitaba la práctica de la actividad física, reportado por los apoderados fueron excluidos del estudio.

\section{Recopilación de datos, sitios de intervención y reclutamiento de participantes}

Todas las evaluaciones y el programa de ejercicios se llevaron a cabo en una Escuela básica de la comuna de Colbún, región del Maule, Chile. El investigador principal del estudio, juntamente con dos profesionales de la actividad física con más de 5 años de experiencia, hicieron todas las evaluaciones. Además, el investigador principal fue el responsable por capacitar a 6 profesores encargados del curso quienes hicieron los ejercicios diarios establecidos en un programa de entrenamiento. Al final de la intervención, los mismos evaluadores recopilaron los resultados de la condición física y índice de masa corporal. Junto con lo anterior, mencionar que los evaluadores y entrenadores fueron ciegos al estudio.

\section{Procedimientos de evaluación}

Para la realización del estudio, se estableció un periodo de inducción a los docentes, a los padres y apoderados, donde se hizo una familiarización con la secuencia de los ejercicios y entrega de información importante sobre la correcta ejecución de los ejercicios que se realizarían y el protocolo de las pruebas físicas. Junto con lo anterior, se entregó el consentimiento informado para su respectiva firma y autorización. Luego, fueron registrados en una planilla los datos de los participantes respecto a la edad, sexo, mediciones antropométricas, flexibilidad, fuerza-resistencia y agilidad. Dicha planilla se utilizó para registrar los datos de dos evaluaciones, la primera antes de iniciar el estudio y la segunda al término de 16 semanas.

\section{Evaluación antropométrica}

Para la evaluación antropométrica fueron considerados protocolos estandarizados (Gordon C, Chumlea W, \& A., 1991; Jordan, 1980), en horario de clases de educación física (a las 10:00h) y realizadas por tres evaluadores profesionales de la actividad física, con conocimiento en el ámbito de evaluaciones antropométricas y físicas, en una sala con la presencia de un apoderado, junto al profesor del curso y de educación física. Fueron realizadas dos evaluaciones (la pri- mera antes de iniciar el estudio y la segunda al término de 16 semanas) considerando los siguientes procedimientos: Evaluación del peso corporal, mediante una balanza digital con precisión de 200 g y una escala de 0 a 150 kilogramos según recomendaciones estandarizadas (Gordon C et al., 1991; Jordan, 1980); Evaluación de la talla, utilizando un estadiómetro de material de aluminio medido en milímetros, con una escala de 0 a 2.50 metros (Gordon C et al., 1991); Evaluación del índice de masa corporal (IMC), considerando la fórmula del índice del peso / talla al cuadrado, la cual fue valorada en la tabla de pediátrica de la Organización Mundial de Salud, OMS (WHO, 2007). Para la clasificación del IMC, valores con más de una desviación típica por encima de la mediana fueron considerados sobrepesos, mientras que valores con más de dos desviaciones típicas por encima de la mediana fueron considerados obesos. Finalmente, valores dentro de la mediana fueron considerados normales en relación a su índice de masa corporal (de Onis \& Lobstein, 2010; WHO, 2020).

\section{Evaluación de la flexibilidad}

Se utilizó la prueba de Wells \& Dillon, con el objetivo de valorar la flexibilidad en la musculatura dorsal de la espalda y posterior del muslo (isquiosurales), siguiendo el protocolo de las pruebas canadienses de evaluación de la aptitud física (Canadian Standardized Test of Fitness)((CSTF), 1986). Antes de la evaluación fue realizado un calentamiento de movilidad articular con una duración de 5 minutos y una familiarización con la ejecución correcta de la prueba de flexibilidad. El material utilizado para esta medición fue un cajón de madera $(30.5 \mathrm{~cm} \times 30.5 \mathrm{~cm} \times 30.5 \mathrm{~cm})$ con una escala de $26 \mathrm{~cm}$ en su extensión, estando el punto cero en el extremo más cercano al evaluado y el $26 \mathrm{~cm}$ coincide con el reposapiés. El evaluado se encontró sin zapatillas y, en posición sentada, tocó sus pies en la caja con las rodillas extendidas. Con hombros flexionados, codos extendidos y manos superpuestas, realizó la flexión del tronco frente a él, que tocó el punto máximo de la escala con sus manos. Se hicieron tres intentos y solo se consideró la mejor marca ((CSTF), 1986; Wells \& Dillon, 1952). Este test fue tomado al inicio del estudio y después de las 16 semanas de intervención.

\section{Evaluación de la fuerza-resistencia}

Se utilizó la prueba chair stand - pararse y sentarse de una silla para evaluar la fuerza-resistencia del tren inferior de acuerdo al protocolo publicado por Jones y Beam et al. (1999)(Jones, Rikli, \& Beam, 1999), tam- 
bién aplicado en la población pediátrica (McKay et al., 2017). Fue realizado un calentamiento utilizando ejercicios de movilidad articular con una duración de 5 minutos y una familiarización considerando tres repeticiones con la ejecución correcta de la prueba de fuerzaresistencia con el objetivo de facilitar la comprensión de lo participante respecto a la forma correcta de realizarlo. Se cuantificó la cantidad de veces «que se levanta y se sienta» el participante en una silla, mediante un cronometrador y fue registrado el mayor número de veces posible durante 30 segundos (Spearman, 1987).

\section{Evaluación de la agilidad}

La agilidad fue evaluada mediante la prueba $4 \times 10 \mathrm{~m}$ shuttle run test que consiste en realizar una carrera y giro a velocidad máxima entre dos líneas paralelas dibujadas en el suelo a 10m de distancia, en la línea de salida se colocó un cono (A) y en la otra línea se colocaron dos conos (B y $\mathrm{C}$ ), cuando se dio la indicación el niño/niña salieron en velocidad a buscar el cono B el cuál lo regresó a la línea de partida tomando el cono A, llevándolo a la ubicación del cono $\mathrm{B}$, luego tomando el cono $\mathrm{C}$ y corriendo rápidamente cruzando la línea de inicio de la carrera. El resultado se midió por medio de un cronómetro, el cual se detuvo al momento que el niño/niña pasó ambos los pies por la línea de inicio, completando 4 carreras. Lo anterior siguiendo las recomendaciones del protocolo de la prueba (Oja \& Jürimäe, 1997). Antes de realizar la prueba los participantes realizaron un calentamiento general con una duración de 5 minutos, luego se explicó el procedimiento y se hizo una prueba de ensayo en la cual el niño solamente realizó el recorrido caminando, los resultados de la prueba se obtuvieron con el mejor tiempo de dos intentos. Dicha prueba presenta alta fiabilidad en la población de escolares (Ramírez-Vélez, Bezerra, Correa-Bautista, Izquierdo, \& Lobelo, 2015) y adolescentes (VicenteRodríguez et al., 2011).

\section{Programa de intervención}

La intervención se realizó en el interior del aula, con una duración total diaria de 30 minutos, distribuida en tres momentos de la jornada escolar: momento número 1 antes de iniciar la primera clase de la mañana (10 minutos), momento número 2 antes de salir al almuerzo (10 minutos) y momento número 3 al término de la jornada (10 minutos), antes de retirarse a sus hogares. Los ejercicios fueron guiados por el profesor correspondiente a la hora señalada de la actividad, previamente capacitado por el investigador principal del estu- dio, en la cual se entregó el conocimiento necesario para desarrollar la actividad en lo relacionado la correcta ejecución, intensidad, duración y pausa de recuperación de los ejercicios. Dichos ejercicios están diseñados para que se realice en un metro cuadrado, no necesita material deportivo extra para su ejecución y se pueden realizar independiente a la vestimenta que tenga el alumno (as), solo se trabaja con su propio cuerpo. Además, todos los ejercicios fueron guiados por una figura grafica la cual se encuentra inserta en un calendario de muro, el cual se instaló en lugar visible en el interior de las salas de clases, este calendario contenía toda la información necesaria para realizar de manera precisa los ejercicios, como es el tiempo de duración, las repeticiones, la intensidad y densidad del ejercicio (ANEXO). El programa tenía un ejercicio distinto cada día, el cual está pensado para la edad y desarrollo del escolar, además, contiene los principios del entrenamiento los cuales son, progresión, especificidad, continuidad, variedad. Finalmente, los ejercicios se realizaron en un metro cuadrado, con intensidad moderada, repeticiones y duración previamente determinada.

\section{Programa}

La siguiente tabla describe los componentes del entrenamiento realizado en el estudio según los principios del entrenamiento de frecuencia, intensidad, tipo, tiempo, volumen y progresión (tabla 1).

Tabla 1

\begin{tabular}{cccccc}
\multicolumn{7}{c}{ Programa de intervención realizado en el estudio } \\
\hline Semanas & Frecuencia & Intensidad & Tipo & Tiempo (diario) & Volumen (semana) \\
\hline 1 & 5 & Leve & Flexibilidad & 30 minutos & 150 minutos \\
2 & 5 & Leve & Resistencia & 30 minutos & 150 minutos \\
3 & 5 & Moderada & Fuerza & 30 minutos & 150 minutos \\
4 & 5 & Moderada & Resistencia & 30 minutos & 150 minutos \\
\hline 5 & 5 & Moderada & Flexibilidad & 30 minutos & 150 minutos \\
6 & 5 & Moderada & Fuerza & 30 minutos & 150 minutos \\
7 & 5 & Moderada & Resistencia & 30 minutos & 150 minutos \\
8 & 5 & Moderada & Fuerza & 30 minutos & 150 minutos \\
\hline 9 & 5 & Moderada & Flexibilidad & 30 minutos & 150 minutos \\
10 & 5 & Moderada & Resistencia & 30 minutos & 150 minutos \\
11 & 5 & Moderada & Fuerza & 30 minutos & 150 minutos \\
12 & 5 & Moderada & Fuerza & 30 minutos & 150 minutos \\
\hline 13 & 5 & Moderada & Flexibilidad & 30 minutos & 150 minutos \\
14 & 5 & Moderada & Resistencia & 30 minutos & 150 minutos \\
15 & 5 & Moderada & Fuerza & 30 minutos & 150 minutos \\
16 & 5 & Moderada & Resistencia & 30 minutos & 150 minutos \\
\hline
\end{tabular}

\section{Análisis de los datos}

Se utilizó el paquete estadístico SPSS (versión 17.0). La prueba de Kolmogorov Smirnov fue utilizada para determinar la normalidad de los datos y aquellos con distribución normal en la comparación intra grupo y entre grupo son expresados como media, desviación estándar (DE) e intervalo de confianza del 95\% (IC del 95\%). Los datos con distribución no normal en la comparación intra grupo y entre grupo fueron expresados por mediana, primer, tercer cuartil, mínimo y máximo. 
La prueba T Student para mediciones independientes fue utilizada para evaluar las posibles diferencias entre grupos pre y post intervención para las variables peso, IMC y fuerza-resistencia, mientras que la prueba no paramétrica correspondiente Mann Whitney fue considerada para las variables estatura, flexibilidad y agilidad. La prueba $T$ Student para mediciones emparejadas fue utilizada para evaluar las posibles diferencias entre grupos pre y post intervención para las variables (grupo niñas: peso, IMC, fuerza-resistencia, agilidad; grupo niños: estatura, peso, IMC, fuerza-resistencia). La prueba no paramétrica correspondiente Wilcoxon, fue utilizada para evaluar diferencias entre grupos pre y post intervención para las variables (grupo niñas: estatura, flexibilidad; grupo niños: flexibilidad, agilidad). El análisis estadístico consideró un nivel de confianza del 95\% y un valor de $\mathrm{p}<0.05$ significativo en todas las pruebas. Además, fue considerado el cálculo del tamaño del efecto utilizando el índice D Cohen para los datos con distribución normal. Un tamaño $>0.80$ fue considerado grande, alrededor de 0.50 moderado y $<0.20$ pequeño (Cohen, 1988). El tamaño de efecto para datos con distribución no normal fue calculado mediante el delta de
Cliff. Cuando se obtiene un valor p significativo, el tamaño del efecto cerca de $+1.0 \mathrm{o}$ «1.0 se considera importante (Macbeth, Razumiejczyk, \& Ledesma, 2011).

\section{Resultados}

Ochenta y cuatro estudiantes fueron reclutados para participar del estudio y entrenados. Sin embargo, 11 no realizaron la última evaluación y fueron excluidos, resultando en un total de setenta y tres estudiantes que participaron de todas las etapas del estudio.

Respecto a las comparaciones de medidas antropométricas y físicas entre genero pre y post intervención no hubo diferencias en la estatura, en el peso, en el índice de masa corporal, en la flexibilidad y en la fuerza-resistencia ( $\mathrm{p}>0.05$ en todas las comparaciones, rango de tamaño de efecto 0 -.35). La agilidad fue mayor en los niños previo (diferencia de 1.64 segundos, $\mathrm{p}<0.01$, delta Cliff $=0.46$ ) y posterior (diferencia de 1 . 45 segundos, $\mathrm{p}<0.01$, delta Cliff $=0.93$ ) a la intervención en comparación con las niñas. Las tablas 2 y 3 presentan las comparaciones entre género previo y posterior a la intervención.

Tabla 2

\begin{tabular}{|c|c|c|c|c|c|}
\hline & Niñas $(n=34)$ & Niños $(n=39)$ & $\mathrm{P}$ valor & $\begin{array}{l}\text { Valor de la } \\
\text { prueba }\end{array}$ & Tamaño de efecto \\
\hline Estatura, $\mathrm{cm}$ & $1.34(1.30 ; 1.35)[1.24 ; 1.49]$ & $1.35(1.30 ; 1.34)[1.24 ; 1.43]$ & 0.185 & $\mathrm{U}=543.500$ & Delta Cliff $=0$ \\
\hline Peso, $\mathrm{Kg}$ & $38.44 \pm 8.09(35.62 ; 41.27)$ & $36.97 \pm 6.86(34.75 ; 39.20)$ & 0.307 & $\mathrm{~T}=.840$ & $\mathrm{~d}=0.20(-.28 ; .64)$ \\
\hline $\mathrm{IMC}, \mathrm{Kg} / \mathrm{cm}^{2}$ & $21.43 \pm 3.16(20.32 ; 22.53)$ & $20.47 \pm 3.36(19.38 ; 21.56)$ & 0.687 & $\mathrm{~T}=1.248$ & $\mathrm{~d}=0.29(-.19 ; .73)$ \\
\hline Flexibilidad de tren inferior, $\mathrm{cm}$ & $-2.00(-6.00 ; 3.50)[-15.00 ; 11.00]$ & $1.00(-5.00 ; 4.00)[-14.00 ; 12.00]$ & 0.622 & $\mathrm{U}=618.500$ & Delta Cliff $=0$ \\
\hline Fuerza-resistencia de tren inferior, repeticiones & $25.79 \pm 4.61(24.18 ; 27.40)$ & $26.79 \pm 3.91(25.52 ; 28.02)$ & 0.260 & $\mathrm{~T}=-1.000$ & $\mathrm{~d}=-0.24(-0.68 ;-0.24)$ \\
\hline Agilidad, segundos & $17.21(15.54 ; 18.62)[13.26 ; 20.52] €$ & $15.57(14.44 ; 17.09)[-13.26 ; 20.52]$ & $<0.001$ & $\mathrm{U}=345.500$ & Delta Cliff $=0.46$ \\
\hline \multicolumn{6}{|c|}{$\begin{array}{l}\text { Abreviaturas: IMC, índice de masa corporal. Los datos son expresados en: mediana (primer; tercer cuartil)[mínimo; máximo], promedio } \pm \text { desviación estándar (límite inferior; límite superior del intervalo de } \\
\text { confianza, IC al 95\%). Delta Cliff: tamaño de efecto para datos no paramétricos. d: d Cohen, tamaño de efecto para datos paramétricos. £: Prueba Wilcoxon. }\end{array}$} \\
\hline \multicolumn{6}{|l|}{ Tabla 3} \\
\hline \multicolumn{6}{|c|}{ Características antropométricas y fisicas de los participantes del estudio posterior a la intervención } \\
\hline & Niñas $(n=34)$ & Niños $(n=39)$ & P valor & $\begin{array}{l}\text { Valor de la } \\
\text { prueba }\end{array}$ & Tamaño de efecto \\
\hline Estatura, $\mathrm{cm}$ & $1.35 \pm 0.06(1.33 ; 1.38)$ & $1.36 \pm 0.04(1.35 ; 1.38)$ & 0.440 & $\mathrm{~T}=-0.776$ & $\mathrm{~d}=-0.02(-0.48 ; 0.44)$ \\
\hline Peso, $\mathrm{Kg}$ & $38.82 \pm 8.41(35.88 ; 41.75)$ & $37.23 \pm 7.15(34.91 ; 39.54)$ & 0.385 & $\mathrm{~T}=0.874$ & $\mathrm{~d}=0.20(-0.27 ; 0.65)$ \\
\hline $\mathrm{IMC}, \mathrm{Kg} / \mathrm{cm}^{2}$ & $20.86 \pm 3.01(19.81 ; 21.92)$ & $19.88 \pm 3.46(18.76 ; 21.01)$ & 0.203 & $\mathrm{~T}=1.284$ & $\mathrm{~d}=0.30(-0.18 ; .074)$ \\
\hline Flexibilidad de tren inferior, $\mathrm{cm}$ & $2.00(-4.00 ; 3.25)[-10.00 ; 11.00]$ & $-2.00(-6.00 ; 2.00)[-13.00 ; 7.00]$ & 0.093 & $\mathrm{U}=511.500$ & Delta Cliff $=0.23$ \\
\hline Fuerza -resistencia de tren inferior, repeticiones & $36.17 \pm 6.91(33.76 ; 38.59)$ & $38.38 \pm 5.81(36.50 ; 40.26)$ & 0.143 & $T=-1.482$ & $\mathrm{~d}=-0.35(-0.78 ; 0.14)$ \\
\hline Agilidad, segundos & $16.49 \pm 1.42(16.00 ; 16.99) *$ & $15.04 \pm 1.67(14.49 ; 15.58)$ & $<0.001$ & $\mathrm{~T}=3.982$ & $\mathrm{~d}=0.93(0.38 ; 1.34)$ \\
\hline
\end{tabular}

Abreviaturas: IMC, índice de masa corporal. Los datos son expresados en: mediana (primer; tercer cuartil)[mínimo; máximo], promedio \pm desviación estándar (límite inferior; límite superior del intervalo de confianza, IC al 95\%). Delta Cliff: tamaño de efecto para datos no paramétricos. d: d Cohen, tamaño de efecto para datos paramétricos. *: Prueba T student.

Tabla 4

Caracteristicas antropométricas y fisicas de niñas que participaron del estudio, previo y posterior al entrenamiento.

\begin{tabular}{cccccc}
\hline & Pre entrenamiento $(\mathrm{n}=34)$ & Post entrenamiento $(\mathrm{n}=34)$ & P valor & Valor de la prueba & Tamaño de efecto \\
\hline Estatura, $\mathrm{cm}$ & $1.34(1.30 ; 1.34)[1.24 ; 1.49]$ & $1.35(1.31 ; 1.35)[1.27 ; 1.51] \in$ & $<0.010$ & $\mathrm{Z}=-3.896$ & $\mathrm{Delta}$ Cliff $=0$ \\
Peso, $\mathrm{Kg}$ & $38.44 \pm 8.09(35.62 ; 41.27)$ & $38.82 \pm 8.41(35.88 ; 41.75)$ & 0.480 & $\mathrm{~T}=-0.715$ & $\mathrm{~d}=-0.05(-0.52 ; 0.43)$ \\
IMC, $\mathrm{Kg} / \mathrm{cm}^{2}$ & $21.43 \pm 3.16(20.32 ; 22.53)$ & $20.86 \pm 3.01(19.81 ; 21.92)^{*}$ & 0.003 & $\mathrm{~T}=3.216$ & $\mathrm{~d}=0.18(-0.31 ; 0.65)$ \\
Flexibilidad de tren inferior, $\mathrm{cm}$ & $-2.00(-6.00 ; 3.50)[-15.00 ; 11.00]$ & $2.00(-4.00 ; 3.25)[-10.00 ; 11.00]$ & 0.234 & $\mathrm{Z}=-1.190$ & $\mathrm{Delta} C \mathrm{Cliff}=-0.11$ \\
Fuerza de tren inferior, repeticiones & $25.79 \pm 4.61(24.18 ; 27.40)$ & $36.17 \pm 6.91(33.76 ; 38.59)^{*}$ & $<0.001$ & $\mathrm{~T}=-9.430$ & $\mathrm{~d}=-1.77(-2.18 ;-1.08)$ \\
Agilidad, segundos & $17.40 \pm 2.10(16.66 ; 18.13)$ & $16.49 \pm 1.42(16.00 ; 16.99)^{*}$ & 0.001 & $\mathrm{~T}=3.487$ & $\mathrm{~d}=0.51(-0.00 ; 0.95)$ \\
\hline
\end{tabular}

Abreviaturas: IMC, índice de masa corporal. Los datos son expresados en: mediana (primer; tercer cuartil)[mínimo; máximo], promedio \pm desviación estándar (límite inferior; límite superior del intervalo de confianza, IC al 95\%). Delta Cliff: tamaño de efecto para datos no paramétricos. d: d Cohen, tamaño de efecto para datos paramétricos. €: Prueba Wilcoxon; *: Prueba T student.

Tabla 5

Caracteristicas antropométricas y fisicas de niños que participaron del estudio, previo y posterior al entrenamien

\begin{tabular}{|c|c|c|c|c|c|}
\hline & Pre entrenamiento $(\mathrm{n}=39)$ & Post entrenamiento $(n=39)$ & P valor & Valor de la prueba & Tamaño de efecto \\
\hline Estatura, $\mathrm{cm}$ & $1.34 \pm 0.05(1.32 ; 1.35)$ & $1.36 \pm 0.04(1.35 ; 1.38)$ & $<0.001$ & $\mathrm{~T}=-12.06$ & $\mathrm{~d}=-0.43(-0.85 ; 0.05)$ \\
\hline Peso, $\mathrm{Kg}$ & $36.97 \pm 6.86(34.75 ; 39.20)$ & $37.23 \pm 7.15(34.91 ; 39.54)$ & 0.460 & $\mathrm{~T}=-0.715$ & $\mathrm{~d}=-0.04(-0.48 ; 0.41)$ \\
\hline $\mathrm{IMC}, \mathrm{Kg} / \mathrm{cm}^{2}$ & $20.47 \pm 3.36(19.38 ; 21.56)$ & $19.88 \pm 3.46(18.76 ; 21.01)^{*}$ & 0.005 & $\mathrm{~T}=2.962$ & $\mathrm{~d}=0.17(-0.28 ; 0.60)$ \\
\hline Flexibilidad de tren inferior, $\mathrm{cm}$ & $1.00(-5.00 ; 4.00)[-14.00 ; 12.00]$ & $-2.00(-6.00 ; 2.00)[-13.00 ; 7.00]$ & 0.109 & $Z=-1.602$ & Delta Cliff $=0.13$ \\
\hline Fuerza de tren inferior, repeticiones & $26.79 \pm 3.91(25.52 ; 28.02)$ & $38.38 \pm 5.81(36.50 ; 40.26) *$ & $<0.001$ & $\mathrm{~T}=-12.150$ & $\mathrm{~d}=-2.34(-2.72 ;-1.60)$ \\
\hline Agilidad, segundos & $15.57(14.44 ; 17.09)[-13.26 ; 20.52]$ & $14.86(13.66 ; 16.03)[-12.80 ; 19.19]$ & 0.002 & $Z=-3.035$ & Delta Cliff $=0.19$ \\
\hline
\end{tabular}

confianza, IC al 95\%). Delta Cliff: tamaño de efecto para datos no paramétricos. d: d Cohen, tamaño de efecto para datos paramétricos. €: Prueba Wilcoxon; *: Prueba T student. 
Respecto a las comparaciones intra gupo, los escolares presentaron una disminución del índice de masa corporal (diferencia de $0.60 \mathrm{~kg} / \mathrm{cm}^{2}$ en ambos géneros), aumento de la fuerza de tren inferior (10.4 repeticiones en niñas y 11.6 en niños) y mejora de la agilidad ( 0.91 segundos en niñas y 0.71 segundos en niños), $\mathrm{p}<.05$ en todas las comparaciones y tamaño de efecto entre 0.20 y 1.77 en niñas y 0.18 y 2.34 en niños. Las tablas 4 y 5 presentan las características antropométricas y físicas intra grupo (niñas y niños), previo y posterior a la intervención.

La figura 2 presenta los grados del índice de masa corporal en niños y niñas. Los escolares redujeron 5.3\% (niños) y 5,9\% (niñas) el grado de obesidad posterior al entrenamiento. Además, cuatro escolares (2 niñas y 2 niños) pasaron del rango obeso para normal y un niño pasó del rango sobrepeso para normal.

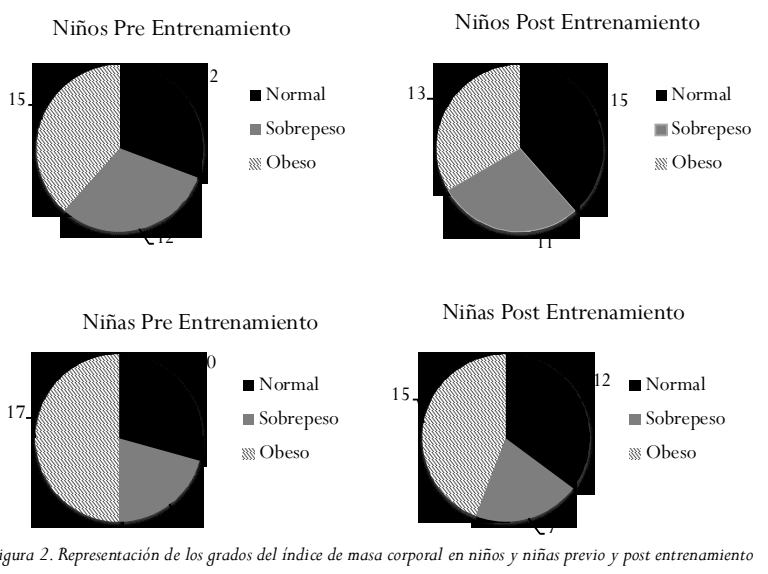

\section{Discusión}

El presente estudio tuvo como objetivo evaluar los efectos de 16 semanas de ejercicios diarios al interior de clases sobre medidas antropométricas y distintas pruebas de rendimiento físico en niños y niñas con edad de 8 a 9 años. Los resultados apuntan una disminución del índice de masa corporal, aumento de fuerza de tren inferior y mayor agilidad, con rango de tamaños de efecto entre 0.17 y 2.34 .

Respecto a la estatura, ambos niños y niñas presentaron un aumento de $1 \mathrm{~cm}$ (niñas) y $2 \mathrm{~cm}$ (niños), con tamaño de efecto moderado (entre 0 y 0.43 ). Según el Ministerio de Salud, el aumento entre 1 y 2 desviaciones estándar positivas puede caracterizar una talla normal alta y alta. Además, el crecimiento mayor a $3.5 \mathrm{~cm}$ en 6 meses podría reflejar en una aceleración de la madurez biológica (MINSAL, 2017). Es importante considerar que no hubo diferencias en el peso y que el índice de masa corporal disminuyó después del entrena- miento en ambos géneros. Por lo tanto, el aumento de la estatura puede reflejar en el menor IMC de los participantes del estudio. Estos resultados corroboran con un estudio previo de entrenamiento realizado por Gunter y colaboradores (2008) realizado tres días a la semana con sesiones de 30 minutos por 7 meses en escolares. Dicho estudio reportó un aumento de la talla de los varones después del entrenamiento de saltos comparado con damas (Gunter et al., 2008). Los resultados del presente estudio no son considerados relevantes debido al bajo tamaño de efecto identificado y a los ejercicios utilizados en el entrenamiento, que no fueron específicos para verificar cambios en esta variable.

En relación al índice de masa corporal, hubo una disminución en ambos géneros después de 16 semanas de ejercicio, sin diferencias entre niñas y niños. Según la literatura, hay evidencia de mejora de la composición corporal de niños que realizan entrenamiento con ejercicios que priorizaron habilidades motoras en combinación con otras intervenciones con período de entrenamiento entre 6 y 60 semanas (Garcia-Hermoso, AlonsoMartinez, Ramirez-Velez, \& Izquierdo, 2020). Sin embargo, es importante considerar la especificidad del ejercicio según el objetivo del entrenamiento. En este contexto, una revisión sistemática que tuvo como objetivo valorar la eficacia de programas de actividades física en el interior escolar, identificó mejorías significantes sobre la composición corporal, el período de entrenamiento de los estudios incluidos fue de 8 semanas hasta 5 años, las estrategias de entrenamiento contenían programas de ejercicios aeróbicos, de fuerza muscular y habilidades motoras, asociados con educación familiar y nutricional (Yuksel, Sahin, Maksimovic, \& Drid, 2020). La relevancia del presente estudio es que fueron identificados cambios en el índice de masa corporal con reducción de $0.60 \mathrm{~kg} / \mathrm{cm}^{2}$ en ambos géneros, con pequeño tamaño de efecto (aproximadamente 0.2) solamente con la intervención de la práctica diaria de actividad física, sin intervenciones asociadas con la educación alimentaria.

En cuanto a la fuerza de tren inferior hubo un aumento significante con tamaño de efecto entre $1.77 \mathrm{y}$ 2.34 en ambos grupos de participantes evaluados. Estudios previos han demostrado que un programa de entrenamiento que involucra distintos tipos de ejercicio, como ejercicios aeróbicos y de fuerza son beneficiosos para aumentar la fuerza muscular de escolares (Engel et al., 2019; Garcia-Hermoso et al., 2020; Yuksel et al., 2020). Sin embargo, solamente un estudio fue realizado en el interior de las escuelas, con una duración prome- 
dio de 6 minutos y 3 veces a la semana con la modalidad de ejercicio utilizando intervalos a altas intensidades (Engel et al., 2019). Es importante considerar que el presente estudio es el primer a considerar la práctica de ejercicios diarias al interior de clases con duración de 30 minutos diarios, utilizando un programa de distintos tipos de ejercicios realizados en $1 \mathrm{~m}^{2}$, sin intervenir en las clases de educación física habituales.

Además, aunque no fueron valorados mediciones respecto a pliegues cutáneos para cuantificar la masa magra, el aumento de la fuerza de tren inferior podría estar asociado a la reducción del índice de masa corporal en los participantes del estudio después de 16 semanas de entrenamiento. Por lo tanto, estos resultados podrían estar asociados a un posible aumento de la masa magra de damas y varones entrenados.

La agilidad de los participantes del estudio fue mayor posterior a la intervención en ambos géneros (diferencia de 0.91 segundos en niñas y 0.71 segundos en niños). Estos hallazgos también son reportados en la literatura después de un programa de entrenamiento con intensidades moderadas (Faigenbaum et al., 2014; Nobre et al., 2017). Aunque estos estudios fueron realizados dentro del interior de las escuelas por 8 semanas (Faigenbaum et al., 2014) y 12 semanas (Nobre et al., 2017), no fueron realizados diariamente. El presente estudio evidenció mejoras en la agilidad de ambos géneros después de 16 semanas. Además, estos resultados pueden estar asociados al aumento de la fuerza muscular del tren inferior observada en ambos grupos de participantes.

En relación al peso de niñas y niños, no hubo diferencias intra y entre grupos posterior al entrenamiento, las niñas obtuvieron un aumento de $0.38 \mathrm{~kg}$ y los niños de $0.26 \mathrm{~kg}$, con bajo tamaño de efecto. Estos resultados están de acuerdo con la mayoría de la literatura, en dónde un aumento entre 0.60 y $0.90 \mathrm{~kg}$ en el peso posterior a un programa de entrenamiento sin orientación nutricional no fueron significantes (Engel et al., 2019; Nobre et al., 2017). Nuestros resultados indican que un programa de ejercicios diarios moderados sin orientaciones o restricciones nutricionales es beneficioso para mantener el peso corporal. Además, es importante considerar que los ejercicios incluidos en el programa de entrenamiento no presentaron una especificidad para el tipo aeróbico que es el más específico para la reducción del peso corporal y porcentaje de grasa (Alves \& Alves, 2019).

Respecto a la flexibilidad, no hubo diferencias entre los participantes del estudio posterior al entrenamien- to. Dichos resultados presentan controversias en la literatura previa. Un estudio de 12 semanas de entrenamiento pliométrico presenta mejora en la flexibilidad corporal en escolares de 7 a 9 años (Nobre et al., 2017). Además, 6 semanas de ejercicios de resistencia muscular y flexibilidad aumentan la flexibilidad corporal de jóvenes con aproximadamente 12 años (Moreira, Akagi, Wun, Moriguchi, \& Sato, 2012). Otro estudio, con ejercicios a intervalos de alta intensidad en el interior escolar no presentó cambios en la flexibilidad corporal (Engel et al., 2019). Considerando las diferencias metodológicas de estos estudios, es importante considerar que la especificidad del entrenamiento es muy importante para observar resultados. Sin embargo, aunque los ejercicios realizados en la presente investigación fueron diversificados, la flexibilidad corporal no presentó mejoría posterior al entrenamiento. Para justificar este resultado, es importante considerar que los ejercicios fueron realizados en un espacio reducido $\left(1 \mathrm{~m}^{2}\right)$, dentro de clase. Además, el trabajo de flexibilidad necesita más espacio para trabajar de forma más amplia las distintas articulaciones, ocupando un mayor tiempo para su ejecución.

Este es el primer estudio latinoamericano que reporta la aplicabilidad de ejercicios físicos diarios de intensidad moderada en el interior del aula en escolares, sin embargo, no está exento de limitaciones. El bajo tamaño de la muestra y la no aleatorización de los participantes deben ser considerados y los resultados deben ser analizados con cautela. Asimismo, el presente estudio servirá como base para futuras investigaciones del tipo ensayo clínico aleatorizado, considerando un grupo control para comparar los resultados y utilizando otras variables cómo la medición del porcentaje de grasa a través de instrumentos no invasivos, la valoración del rendimiento académico y el control nutricional en conjunto con el programa de ejercicios.

\section{Conclusiones}

Un programa de entrenamiento diario con una intensidad, frecuencia y volumen establecidos, sin implementación deportiva, en espacios reducidos para la ejecución de los ejercicios, que se puede realizar en el interior de la sala de clase disminuye el índice de masa corporal. Además, presenta una importante mejoría en el rendimiento, considerando incremento en la fuerza muscular y agilidad de escolares entre 8 y 9 años de ambos géneros. Los cambios encontrados fueron representativos con moderados a altos tamaños de efecto 
y solamente fueron a través de la actividad física, sin recomendación para mantener hábitos alimentarios saludables o cualquier otro tipo de orientación nutricional.

\section{Referencias}

(CSTF), C. S.T. o. F. (1986). Operations manual. Ottawa: Minister of State.

Al-Muhaimeed, A. A., Dandash, K., Ismail, M. S., \& Saquib, N. (2015). Prevalence and correlates of overweight status among Saudi school children. Ann Saudi Med, 35(4), 275-281. doi: 10.5144/02564947.2015.275

Alves, J. G. B., \& Alves, G. V. (2019). Effects of physical activity on children's growth. J Pediatr (Rio J), 95 Suppl 1, 72-78. doi: 10.1016/j.jped.2018.11.003

Bärnighausen, T., Røttingen, J. A., Rockers, P., Shemilt, I., \& Tugwell, P. (2017). Quasi-experimental study designs series-paper 1: introduction: two historical lineages. J Clin Epidemiol, 89, 4-11. doi: 10.1016/ j.jclinepi.2017.02.020

Bornstein, M. H., Jager, J., \& Putnick, D. L. (2013). Sampling in Developmental Science: Situations, Shortcomings, Solutions, and Standards. Dev Rev, 33(4), 357-370. doi: 10.1016/j.dr.2013.08.003

Carsley, S., Tu, K., Parkin, P., Pullenayegum, E., \& Birken, C. (2018). Overweight and obesity in preschool aged children and risk of mental health service utilization. Int J Obes (Lond). doi: 10.1038/ s41366-018-0280-1

Carson, V., Hunter, S., Kuzik, N., Gray, C. E., Poitras, V. J., Chaput, J. P., . . . Tremblay, M. S. (2016). Systematic review of sedentary behaviour and health indicators in school-aged children and youth: an update. Appl Physiol Nutr Metab, 41 (6 Suppl 3), S240265. doi: 10.1139/apnm-2015-0630

Cohen, J. (1988). The concepts of power analysis. Statistical power analysis for the behavioral sciences. New Brunswick, NJ.

Cruz, T. H., Davis, S. M., Myers, O. B., O’Donald, E. R., Sanders, S. G., \& Sheche, J. N. (2016). Effects of an Obesity Prevention Intervention on Physical Activity Among Preschool Children: The CHILE Study. Health Promot Pract, 17(5), 693-701. doi: $10.1177 / 1524839916629974$

de Onis, M., \& Lobstein, T. (2010). Defining obesity risk status in the general childhood population: which cut-offs should we use? Int J Pediatr Obes, 5(6), 458460. doi: 10.3109/17477161003615583

Engel, F. A., Wagner, M. O., Schelhorn, F., Deubert, F.,
Leutzsch, S., Stolz, A., \& Sperlich, B. (2019). Classroom-Based Micro-Sessions of Functional HighIntensity Circuit Training Enhances Functional Strength but Not Cardiorespiratory Fitness in School Children-A Feasibility Study. Front Public Health, 7, 291. doi: 10.3389/fpubh.2019.00291

Faigenbaum, A. D., Myer, G. D., Farrell, A., Radler, T., Fabiano, M., Kang, J., . . . Hewett, T. E. (2014). Integrative neuromuscular training and sex-specific fitness performance in 7-year-old children: an exploratory investigation. JAthlTrain, 49(2), 145-153. doi: 10.4085/1062-6050-49.1.08

Garcia-Hermoso,A.,Alonso-Martinez, A. M., RamirezVelez, R., \& Izquierdo, M. (2020). Effects of Exercise Intervention on Health-Related Physical Fitness and Blood Pressure in Preschool Children: A Systematic Review and Meta-Analysis of Randomized Controlled Trials. Sports Med, 50(1), 187-203. doi: 10.1007/s40279-019-01191-w

Giakoni, F., Bettancourt, P. P., \& Duclos-Bastías, D. (2021). Educación Física en Chile: tiempo de dedicación y su influencia en la condición física, composición corporal y nivel de actividad física en escolares. $\operatorname{Retos}(39), 24-29$.

Gordon C, ChumleaW, \& A., R. (1991). En: Martorell, Reynaldo., Lohman, Timothy G., Roche, Alex F. Anthropometric standardization reference manual. Champaign [Ill.]: Human Kinetics Books.

Gunter, K., Baxter-Jones, A. D., Mirwald, R. L., Almstedt, H., Fuchs, R. K., Durski, S., \& Snow, C. (2008). Impact exercise increases BMC during growth: an 8-year longitudinal study. J Bone Miner Res, 23(7), 986-993. doi: 10.1359/jbmr.071201

Hollis, J. L., Sutherland, R., Williams, A. J., Campbell, E., Nathan, N., Wolfenden, L., . . Wiggers, J. (2017). A systematic review and meta-analysis of moderateto-vigorous physical activity levels in secondary school physical education lessons. Int $J$ Behav Nutr PhysAct, 14(1), 52. doi: 10.1186/s12966-017-0504-0

Jones, C. J., Rikli, R. E., \& Beam, W. C. (1999). A 30-s chair-stand test as a measure of lower body strength in community-residing older adults. Res Q Exerc Sport, 70(2), 113-119. doi: 10.1080/ 02701367.1999.10608028

Jordan, R. (1980). Crecimiento y desarrollo del niño de Cuba. Bol Med Hosp Inf México, 37.

Macbeth, G., Razumiejczyk, E., \& Ledesma, R. D. (2011). Cliff's Delta Calculator: A non-parametric effect size program for two groups of observations. Universitas Psychologica, 10, 545-555. 
McKay, M. J., Baldwin, J. N., Ferreira, P., Simic, M., Vanicek, N., \& Burns, J. (2017). Reference values for developing responsive functional outcome measures across the lifespan. Neurology, 88(16), 15121519. doi: $10.1212 /$ wnl.0000000000003847

MINSAL. (2017). Patrones de crecimiento para la evaluación nutricional de niños, niñas y adolescentes desde el nacimiento hasta los 19 años de edad. Santiago, Chile.

Mönckeberg, B. F., \& Muzzo, B. F. (2015). La desconcertante epidemia de obesidad. Revista chilena de nutrición, 42, 96-102.

Moreira, R. F., Akagi, F. H., Wun, P.Y., Moriguchi, C. S., \& Sato, T. O. (2012). Effects of a school based exercise program on children's resistance and flexibility. Work, 41 Suppl 1, 922-928. doi: 10.3233/ wor-2012-0264-922

Nobre, G. G., de Almeida, M. B., Nobre, I. G., Dos Santos, F. K., Brinco, R. A., Arruda-Lima, T. R., . . . Moura-Dos-Santos, M. A. (2017). Twelve Weeks of Plyometric Training Improves Motor Performance of 7- to 9-Year-Old Boys Who Were Overweight/ Obese: A Randomized Controlled Intervention. J Strength Cond Res, 31(8), 2091-2099. doi: 10.1519/ jsc.0000000000001684

Oja, L., \& Jürimäe, T. (1997). Assessment of motor ability of 4- and 5-year-old children. Am J Hum Biol, 9(5), 659-664. doi: 10.1002/(sici)15206300(1997)9:5<659::aid-ajhb12>3.0.co;2-1

Ramírez-Vélez, R., Bezerra, D., Correa-Bautista, J., Izquierdo, M., \& Lobelo, F. (2015). Reliability of Health-Related Physical Fitness Tests among Colombian Children and Adolescents: The FUPRECOL Study. PLoS One, 10. doi: 10.1371/ journal.pone. 0140875

Rodríguez-Fernández, J. E., Rico-Díaz, J., Neira-Martín, P. J., \& Navarro-Patón, R. (2021). Actividad física realizada por escolares españoles según edad y género. Retos(39), 238-245.
Rosa Guillamón, A., Garcia Canto, E., \& Jose Carrillo López, P. (2019). Capacidad aeróbica y rendimiento académico en escolares de educación primaria. Re$\operatorname{tos}(35), 351-354$.

Spearman, C. (1987). The proof and measurement of association between two things. Am.J.Psychol., 100(34), 441-471.

Tambalis, K. D., Panagiotakos, D. B., Psarra, G., \& Sidossis, L. S. (2018). Insufficient Sleep Duration Is Associated With Dietary Habits, Screen Time, and Obesity in Children. J Clin Sleep Med, 14(10), 16891696. doi: 10.5664/jcsm.7374

Vicente-Rodríguez, G., Rey López, J., Ruiz, J., JiménezPavón, D., Bergman, P., Ciarapica, D., ... Ortega, F. (2011). Interrater Reliability and Time Measurement Validity of Speed-Agility Field Tests in Adolescents. Journal of strength and conditioning research / National Strength \& Conditioning Association, 25, 2059-2063. doi: 10.1519/JSC.0b013e3181e742fe Wells, K. F., \& Dillon, E. K. (1952). The Sit and ReachA Test of Back and Leg Flexibility. Research Quarterly. American Association for Health, Physical Education and Recreation, 23(1), 115-118. doi: 10.1080/ 10671188.1952.10761965

WHO. (2007). Growth reference 5-19 years. World Health Organization.

WHO. (2016). Informe de la Comisión para acabar con la obesidad infantil.

WHO. (2020). Growth reference 5-19 years. from World Healthy Organization https:// www.who.int/growthref/who2007_bmi_for_age/ en/

Yuksel, H. S., Sahin, F. N., Maksimovic, N., \& Drid, P. (2020). School-Based Intervention Programs for Preventing Obesity and Promoting Physical Activity and Fitness: A Systematic Review. Int J Environ Res Public Health., 17(1). doi: 10.3390/ijerph17010347

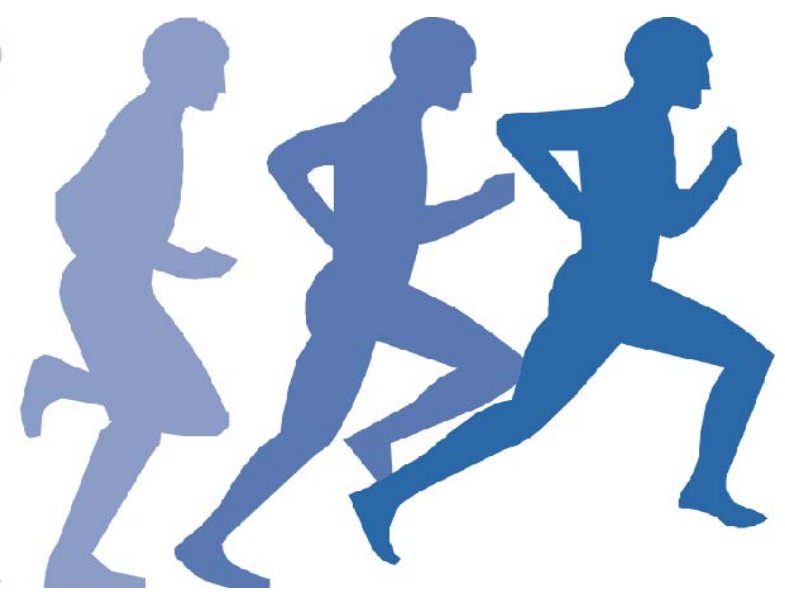

